\title{
Association Between HMGB1 and Thrombogenesis in a Hyperlipaemia-induced Microminipig Model of Atherosclerosis
}

\author{
SATORU KAKE ${ }^{1,2^{*}}$, HIROAKI KAWAGUCHI ${ }^{3 *}$, TOMOKA NAGASATO ${ }^{4,5}$, TOMONOBU YAMADA ${ }^{6}$, \\ TAKASHI ITO ${ }^{4}$, IKURO MARUYAMA ${ }^{4}$, NAOKI MIURA ${ }^{7}$ and AKIHIDE TANIMOTO ${ }^{8}$ \\ ${ }^{1}$ Laboratory of Veterinary Pharmacology, Joint Faculty of Veterinary Medicine, \\ Yamaguchi University, Yamaguchi, Japan; \\ ${ }^{2}$ Department of Comparative Animal Science, College of Life Science, \\ Kurashiki University of Science and The Arts, Okayama, Japan; \\ ${ }^{3}$ Hygiene and Health Promotion Medicine, Graduate School of Medical and Dental Sciences, \\ Kagoshima University, Kagoshima, Japan; \\ ${ }^{4}$ Systems Biology in Thromboregulation, Graduate School of Medical and Dental Sciences, \\ Kagoshima University, Kagoshima, Japan; \\ ${ }^{5}$ Research Institute, Fujimori Kogyo Co., Ltd., Kanagawa, Japan; \\ ${ }^{6}$ Drug Safety Research Laboratories, Shin Nippon Biomedical Laboratories, Ltd., Kagoshima, Japan; \\ ${ }^{7}$ Veterinary Teaching Hospital, Joint faculty of Veterinary Medicine, Kagoshima University, Kagoshima, Japan; \\ ${ }^{8}$ Department of Molecular and Cellular Pathology, \\ Graduate School of Medical and Dental Sciences, Kagoshima University, Kagoshima, Japan
}

\begin{abstract}
Background/Aim: An appropriate animal model is essential to investigate the relationship between inflammation, atherosclerosis, and thrombogenesis, and the development of preventive measures and therapies for atherosclerosis. Materials and Methods: Atherosclerosis was induced in Microminipigs (MMPs) using a high-fat diet. We assessed high mobility group box 1 (HMGB1) expression levels and measured thrombus formation using a Total Thrombus Formation Analysis System (T-TAS). MMPs were divided into a normal diet (control) group and four high-fat diet groups, with differing amounts of cholesterol. After 8 weeks, blood was collected for analysis. Results: HMGB1 levels increased with increasing dietary cholesterol, and a
\end{abstract}

This article is freely accessible online.

*These Authors contributed equally to this study.

Correspondence to: Naoki Miura, Veterinary Teaching Hospital, Joint faculty of Veterinary Medicine, Kagoshima University, Kagoshima, 890-0065, Japan. Tel: +81 992853527, Fax: +81 992858751, e-mail: k9236024@kadai.jp; Akihide Tanimoto, Department of Molecular and Cellular Pathology, Kagoshima University Graduate School of Medical and Dental Sciences, 8-351, Sakuragaoka, Kagoshima 890-8544, Japan. Tel: +81 992755263 , Fax: +819922646348, e-mail: akit09@m3.kufm.kagoshima-u.ac.jp

Key Words: HMGB1, thrombus formation, Microminipig. negative correlation was found between HMGB1 levels and thrombus formation time. Conclusion: T-TAS is useful in the assessment of thrombogenesis in MMPs and HMGB1 is associated with thrombus formation.

Atherosclerosis is a progressive disease characterized by thickening of the arterial walls due to an increase in substances such as fat and cholesterol. Macrophages and downstream signalling pathways play an important role in plaque formation. Atherosclerosis is a multifactorial genetic disease associated with a number of environmental factors. Common risk factors include dyslipidaemias such as hypercholesterolaemia (1). It has also been suggested that atherosclerosis can be triggered by local angiitis caused by, for example, bacterial or viral infections (2). Therefore, research often focusses on atherosclerosis as a chronic inflammatory disease. One of the inflammatory factors associated with atherosclerosis is high mobility group box 1 (HMGB1), a protein secreted from activated macrophages following damage and infection. HMGB1 acts as a damage-associated molecular pattern (DAMP), activating the inflammasome to produce and release inflammatory cytokines such as IL- $1 \beta$ and TNF $\alpha$, thereby promoting inflammation (3). HMGB1 has also attracted attention for its effects on coagulation, promoting atherosclerosis by inducing tissue factors and haemostatic reactions (4). Thus, progression of atherosclerosis can restrict or block arterial blood flow leading to cardiovascular or cerebrovascular disease. However, the association between 


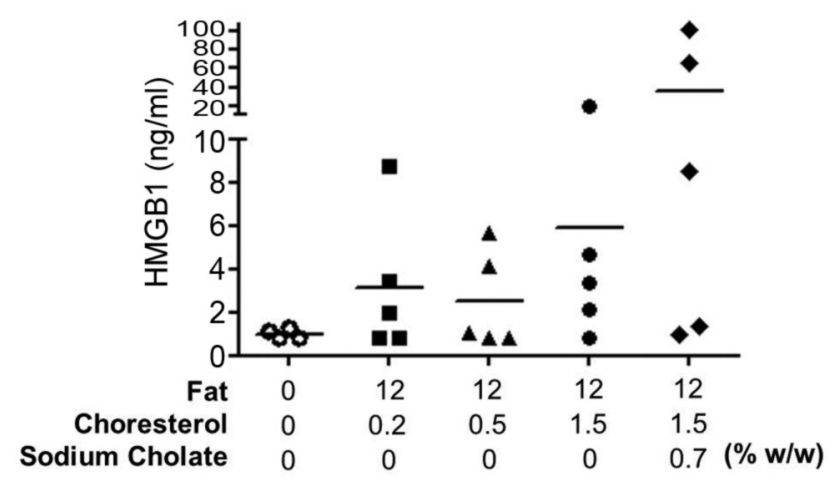

Figure 1. Serum HMGB1 in the control and cholesterol-fed groups.

inflammation and thrombosis in atherosclerosis has not been fully elucidated. Identification of the underlying causes of atherosclerosis is important to establish prevention measures and therapies for the disease.

As early-stage atherosclerosis is asymptomatic, it is difficult to assess and study therapeutic effects in patients before the disease has progressed. Animal models are therefore essential to assess efficacy of therapies in early stages of the disease (5). However, besides the ethical and welfare issues in relation to animal experiments, characteristics of conventional atherosclerosis models are different from those of humans, e.g. low-density lipoprotein (LDL) cholesterol distribution (6-8). In an aim to improve animal models of atherosclerosis, attention has been directed towards the Microminipig (MMP) $(9,10)$.

In MMPs, white thrombus formation time measured by Total Thrombosis Formation Analysis System (T-TAS) was found to be shorter than in humans, suggesting MMPs have a strong thrombotic tendency (11). As for lipid metabolism, by examination of their cholesterol fractionation, MMPs were shown to have high LDL cholesterol levels, as observed in humans and domesticated pigs (12). Hypercholesterolaemia and atherosclerotic lesions have been shown to be induced in MMPs fed with high-fat and high-cholesterol diet (12\% fat, $5 \%$ cholesterol, $0.7 \%$ sodium cholate) for 12 weeks $(13,14)$.

The present study examined the association between thrombogenesis and HMGB1, by inducing atherosclerosis in MMPs using different amounts of dietary cholesterol.

\section{Materials and Methods}

Four-month-old male MMPs (Fuji Micra Inc., Shizuoka, Japan) were fed in an air-conditioned room at Kagoshima University. The room was constantly kept at $24 \pm 3^{\circ} \mathrm{C}, 50 \pm 20 \%$ relative humidity with a $12 \mathrm{~h} \mathrm{light/dark} \mathrm{cycle.} \mathrm{Water} \mathrm{was} \mathrm{freely} \mathrm{available.} \mathrm{Twenty-}$ five pigs were randomly divided into five groups with five pigs in each group. The control group was fed a normal diet (ND, Kodakara

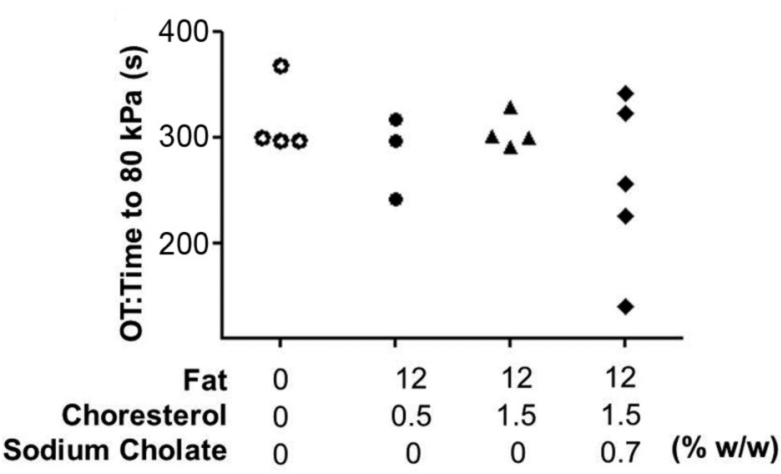

Figure 2. White thrombosis formation assessed by T-TAS. White thrombosis formation was assessed by the occlusion time (OT). Shorter time indicates more thrombosis tendency.

73; Marubeni Nisshin Feed Inc., Tokyo, Japan), comprising less than $9.0 \% \mathrm{w} / \mathrm{w}$ carbohydrate, greater than $15.0 \% \mathrm{w} / \mathrm{w}$ protein, greater than $2.0 \% \mathrm{w} / \mathrm{w}$ fat, and less than $9.0 \%$ fibre. Experimental groups were fed a high-fat diet (refined lard, 12\% w/w; Miyoshi Oil \& Fat Co., Ltd., Tokyo, Japan) and each group received different amounts of cholesterol (Wako Pure Chemical Industries, Ltd. Osaka, Japan): Low (0.2\% w/w), Mid (0.5\% w/w), High (1.5\% w/w), and High Dose dissolved in sodium cholate (SC, $0.7 \%$ w/w; Wako Pure Chemical Industries, Ltd). All groups were fed quantities equivalent to $1.6-3 \%$ of their weight, once, every morning for 8 weeks. The use of animals in this research complied with all relevant guidelines set by Kagoshima University.

After 8 weeks, blood was collected from the MMPs via the cranial vena cava.

Serum concentrations of HMGB1 were assessed using a HMGB1 ELISA Kit II (Shino-test, Tokyo, Japan), according to the manufacturer's instructions.

White thrombus formation assays were performed using a T-TAS (Fujimori Kogyo Co., Ltd., Tokyo, Japan). Blood was collected into a tube containing $3.2 \%$ sodium citrate, and mixed with $20 \mu \mathrm{l} 0.3 \mathrm{M}$ $\mathrm{CaCl}_{2}$ containing $1.25 \mathrm{mg} / \mathrm{ml}$ corn trypsin inhibitor immediately before application to the microchip. The solution was perfused over a microchip capillary coated with collagen and tissue thromboplastin at a flow rate of $10 \mu \mathrm{l} / \mathrm{min}$. The white thrombus formation process was monitored by flow pressure changes in the capillary with a pressure sensor. As white thrombus formation spread on the coated surface, the capillary was gradually occluded, increasing the flow pressure. We calculated the lag time for the flow pressure to increase by $80 \mathrm{kPa}$ (T80), representing almost complete occlusion of the capillary.

For statistical analysis, one-way analysis of variance (one-way ANOVA) was performed to test differences between three or more groups. Correlation analysis was performed using Spearman's correlation coefficient. Prism 7.0 and JMP13.0 were used to perform the statistical tests and $p<0.05$ was determined as significant.

\section{Results}

Serum HMGB1 levels of the control group and cholesterolfed groups are shown in Figure 1. There were individual MMPs with increased HMGB1 levels in Low, Mid, High, and High+SC groups, and the levels increased with 


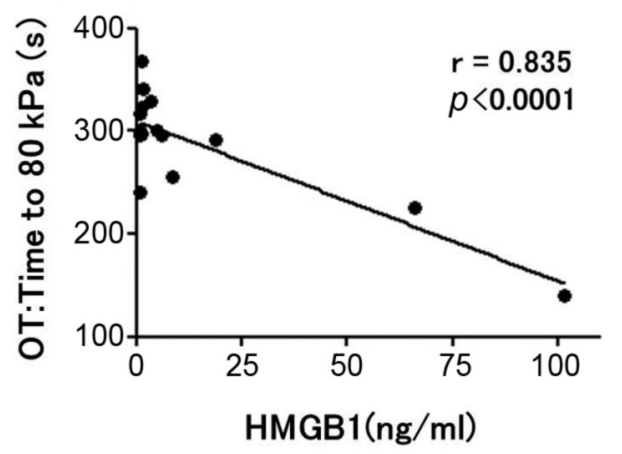

Figure 3. Correlation between HMGB1 concentration and white thrombosis formation time. Thrombosis formation and HMGB1 levels were significantly correlated.

increasing cholesterol in the diet. None of the MMPs in the control group showed an increase in HMGB1 levels. In contrast, an extreme increase in HMGB1 was observed in two MMPs in the High+SC group.

Figure 2 shows the changes in white thrombus formation as assessed by T-TAS. Although there were no significant differences when compared to control, there were individual MMPs exhibiting a shorter white thrombus formation time in the Mid and High+SC groups.

The correlation between white thrombus formation and HMGB1 levels was also investigated. Figure 3 shows white thrombus formation in the blood of individual MMPs that exhibited an increase in HMGB1 levels under cholesterol loading. A concentration-dependent obstruction of blood flow was observed with a significant negative correlation between HMGB1 concentration and white thrombus formation time $(r=0.835, p<0.0001)$.

\section{Discussion}

To the best of our knowledge, this is the first report to show the association between HMGB1 and thrombogenesis using an animal model of atherosclerosis. MMPs are a useful model to study the pathogenesis of atherosclerosis, as MMP atherosclerotic lesions are pathologically and pathophysiologically similar to those of humans, and lesions can be induced in a short period of time.

HMGB1 is a nuclear non-histone DNA-binding protein, which stabilizes nucleosome formation. HMGB1 plays an essential role in the expression of transcription factors such as $\mathrm{p} 53$ and NF-kB $(15,16)$. It also serves as a cytokine when released by activated mononuclear phagocytes and necrotic cells, triggering inflammatory reactions and immune responses $(3,17-19)$. Locally, chronic HMGB 1 can cause atherosclerosis. HMGB1 released from cells can trigger release of inflammatory cytokines such as IL-1 $\beta$ and TNF $\alpha$, promoting inflammation (3). HMGB1 is also thought to play a role in atherosclerosis through thrombus formation, and has been shown to induce tissue factors and promote haemostatic reactions involved in coagulation (4). Given a recent study that identified overexpression of HMGB 1 in atherosclerosis lesions in humans (20), our observation of an association between HMGB1 and increased thrombosis in atherosclerotic MMPs could be relevant to patients.

While atherosclerosis is known to promote hypertension, hyperglycaemia, and hyperlipidaemia, identification of early signs such as increased serum LDL cholesterol and HMGB1 levels are useful to prevent progression of atherosclerosis in asymptomatic patients. A previous study has reported that foam cells (monocytes in the vascular intima which have englobed LDL cholesterol) and free cholesterol form an atheromatous plaque, and monocytes aggregated in the plaque overexpress HMGB1 (21). In line with this, we observed that serum HMGB1 levels increased with cholesterol loading in MMPs, suggesting a link between cholesterol and HMGB1 and highlighting the possibility of detecting early-stage atherosclerosis by measuring HMGB1 levels. Furthermore, from the T-TAS data, blood with increased HMGB1 levels from cholesterol loading had a shorter time to occlusion of the capillary, meaning increased thrombogenesis. This also suggests that HMGB1 is associated with one of the thrombus-inducing factors in atherosclerotic plaque formation. Limitations of the study include that the increase in HMGB1 was only observed in a number of the cholesterol fed MMPs. This may be because of the short time period of observation. We were also unable to assess existing thrombi in the MMPs, because we observed the living animal model in this study. Further study is necessary to alleviate these issues and confirm the findings.

\section{Conflicts of Interest}

TN is an employee of Fujimori Kogyo Co., Ltd.

\section{Authors' Contributions}

Study conception and design, IM, NM, AT; experiments and data acquisition: SK, HK, TN, TY, NM; data analysis and interpretation: HK, TI, NM; draft writing: SK, NM; review and revision: HK, TI, IM, NM, AT.

\section{Acknowledgements}

This work was partly supported by Health Labour Sciences Research Grant (no. 33361105) from the Ministry of Health, Labour and Welfare of Japan (to NM, HK, and AT), Adaptable and Seamless Technology transfer Program (A-Step No. AS2316907E) from the Ministry of Education Culture, Sports, Science and Technology of Japan (to AT and HK), Suzuken Memorial 
Foundation (to NM, HK and AT) and SENSHIN Medical Research Foundation (to NM, HK and AT). The Authors are grateful to Mr. T. Motokado (SNBL, Ltd.), Dr. T. Nishimura, and Mr. N. Kaneko (Fuji Micra Inc.) for their advice and valuable technical assistance. The Authors thank Hanne Gadeberg, PhD, from Edanz Group (https://en-author-services.edanzgroup.com/) for editing a draft of this manuscript.

\section{References}

1 Wouters K, Shiri-Sverdlov R, van Gorp PJ, van Bilsen M and Hofker MH: Understanding hyperlipidemia and atherosclerosis: lessons from genetically modified apoe and ldlr mice. Clin Chem Lab Med 43: 470-479, 2005. PMID: 15899668. DOI: 10.1515/CCLM.2005.085

2 Campbell LA and Rosenfeld ME: Infection and atherosclerosis development. Arch Med Res 46: 339-350, 2015. PMID: 26004263. DOI: $10.1016 /$ j.arcmed.2015.05.006

3 Lu B, Wang C, Wang M, Li W, Chen F, Tracey KJ and Wang H: Molecular mechanism and therapeutic modulation of high mobility group box 1 release and action: an updated review. Expert Rev Clin Immunol 10: 713-727, 2014. PMID: 24746113. DOI: $10.1586 / 1744666 X .2014 .909730$

4 Ito $\mathrm{T}$ and Maruyama I: Thrombomodulin: protectorate God of the vasculature in thrombosis and inflammation. J Thromb Haemost 1: 168-173, 2011. PMID: 21781252. DOI: 10.1111/ j.1538-7836.2011.04319

5 Turk JR, Henderson KK, Vanvickle GD, Watkins J and Laughlin $\mathrm{MH}$ : Arterial endothelial function in a porcine model of early stage atherosclerotic vascular disease. Int J Exp Pathol 86: 335345, 2005. PMID: 16191105. DOI: 10.1111/j.0959-9673. 2005.00446

6 Reddick RL, Zhang SH and Maeda N: Atherosclerosis in mice lacking apo E. Evaluation of lesional development and progression. Arterioscler Thromb 14: 141-147, 1994. PMID: 8274470. DOI: 10.1161/01.atv.14.1.141

7 Shiomi M, Ito $\mathrm{T}$, Tsukada $\mathrm{T}$, Yata $\mathrm{T}$ and Ueda M: Cell compositions of coronary and aortic atherosclerotic lesions in WHHL rabbits differ. An immunohistochemical study. Arterioscler Thromb 14: 931-937, 1994. PMID: 8199184. DOI: 10.1161/01 atv.14.6.931

8 Schwartz SM, Galis ZS, Rosenfeld ME and Falk E: Plaque rupture in humans and mice. Arterioscler Thromb Vasc Biol 27: 705-713, 2007. PMID: 17332493. DOI: 10.1161/01.ATV. 0000261709.34878 .20

9 Kaneko N, Itoh K, Sugiyama A and Izumi Y: Microminipig, a non-rodent experimental animal optimized for life science research: preface. J Pharmacol Sci 115: 112-114, 2011. PMID: 21258171. DOI: $10.1254 /$ jphs.10r16fm

10 Kawaguchi H, Yamada T, Miura N, Takahashi Y, Yoshikawa T, Izumi H, Kawarasaki T, Miyoshi N and Tanimoto A: Reference values of hematological and biochemical parameters for the world smallest microminipigs. J Vet Med Sci 74: 933-936, 2012. PMID: 22362255. DOI: 10.1292/jvms.11-0571

11 Miura N, Kawaguchi H, Nagasato T, Yamada T, Ito T, Izumi H, Shameshima H, Miyoshi N, Tanimoto A and Maruyama I: Coagulation activity and white thrombus formation in the microminipig. In Vivo 27: 357-361, 2013. PMID: 23606691.
12 Kawaguchi H, Yamada T, Miura N, Noguchi M, Izumi H, Miyoshi $\mathrm{N}$ and Tanimoto A: Sex differences of serum lipid profile in novel microminipigs. In Vivo 27: 617-621, 2013. PMID: 23988896.

13 Miyoshi N, Horiuchi M, Inokuchi Y, Miyamoto Y, Miura N, Tokunaga S, Fujiki M, Izumi Y, Miyajima H, Nagata R, Misumi $\mathrm{K}$, Takeuchi T, Tanimoto A, Yasuda N, Yoshida $\mathrm{H}$ and Kawaguchi H: Novel microminipig model of atherosclerosis by high fat and highcholesterol diet, established in Japan. In Vivo 24: 671-680, 2010. PMID: 20952732.

14 Kawaguchi H, Yamada T, Miura N, Ayaori M, Uto-Kondo H, Ikegawa M, Noguchi M, Wang KY, Izumi $\mathrm{H}$ and Tanimoto A: Rapid development of atherosclerosis in the world's smallest Microminipig fed a high-fat/high-cholesterol diet. J Atheroscler Thromb 21: 186-203, 2014. PMID: 24257467. DOI: 10.5551/ jat.21246

15 Andersson U, Erlandsson-Harris $\mathrm{H}$, Yang $\mathrm{H}$ and Tracey KJ: HMGB1 as a DNA-binding cytokine. J Leukoc Biol 72: 10841091, 2002. PMID: 12488489.

16 Müller S, Ronfani L and Bianchi ME: Regulated expression and subcellular localization of HMGB1, a chromatin protein with a cytokine function. J Intern Med 255: 332-343, 2004. PMID: 14871457. DOI: $10.1111 /$ j.1365-2796.2003.01296

17 Harris HE and Raucci A: Alarmin(g) news about danger: Workshop on Innate Danger Signals and HMGB1. EMBO Rep 7: 774-778, 2006. PMID: 16858429. DOI: 10.1038/sj.embor. 7400759

18 Scaffidi P, Misteli T and Bianchi ME: Release of chromatin protein HMGB1 by necrotic cells triggers inflammation. Nature 418: 191-195, 2002. PMID: 12110890. DOI: 10.1038/ nature 00858

19 Palumbo R, Sampaolesi M, De Marchis F, Tonlorenzi R, Colombetti S, Mondino A, Cossu G and BianchiME: Extracellular HMGB1, a signal of tissue damage, induces mesoangioblast migration and proliferation. J Cell Biol 164: 441-449, 2004. PMID: 14744997. DOI: 10.1083/jcb.200304135

20 Kalinina N, Agrotis A, Antropova Y, DiVitto G, Kanellakis P, Kostolias G, Ilyinskaya O, Tararak E and Bobik A: Increased expression of the DNA-binding cytokine HMGB1 in human atherosclerotic lesions: role of activated macrophages and cytokines. Arterioscler Thromb Vasc Biol 24: 2320-2325, 2004. PMID: 15374849. DOI: 10.1161/01.ATV.0000145573.36113.8a

21 Kanellakis P, Agrotis A, Kyaw TS, Koulis C, Ahrens I, Mori S, Takahashi HK, Liu K, Peter K, Nishibori M and Bobik A: Highmobility group box protein 1 neutralization reduces development of diet-induced atherosclerosis in apolipoprotein e-deficient mice. Arterioscler Thromb Vasc Biol 31: 313-319, 2011. PMID: 21088249. DOI: 10.1161/ATVBAHA.110.218669

Received March 21, 2020

Revised March 31, 2020 Accepted April 1, 2020 